\title{
Graphene-induced unique polarization tuning properties of excessively tilted fiber grating
}

\author{
Biqiang Jiang, ${ }^{1,2}$ Guolu Yin, ${ }^{2}$ Kaiming Zhou, ${ }^{2,3}$ Changle Wang, ${ }^{2}$ Xuetao \\ GAN, ${ }^{1}$ JIANLIN ZHAO, ${ }^{1}$ AND LIN ZHANG ${ }^{2}{ }^{*}$ \\ ${ }^{1}$ Key Laboratory of Space Applied Physics and Chemistry, Ministry of Education, and the Shaanxi Key Laboratory of Optical Information \\ Technology, School of Science, Northwestern Polytechnical University, Xi'an 710072, China \\ ${ }^{2}$ Aston Institute of Photonic Technologies, Aston University, Birmingham B4 7ET, U.K. \\ ${ }^{3}$ State Key Laboratory of Transient Optics and Photonics, Xi'an Institute of Optics and Precision Mechanics, Chinese Academy of Sciences, Xi'an 710119, China \\ *Corresponding author: I.zhang@aston.ac.uk
}

Received XX Month XXXX; revised XX Month, XXXX; accepted XX Month XXXX; posted XX Month XXXX (Doc. ID XXXXX); published XX Month XXXX

By exploiting the polarization-sensitive coupling effect of graphene with the optical mode, we investigate the polarization modulation properties of a hybrid waveguide of graphene-integrated excessively tilted fiber grating (Ex-TFG). The theoretical analysis and experimental results demonstrate that the real and imaginary parts of complex refractive index of few-layer graphene exhibit different effects on transverse electric (TE) and transverse magnetic (TM) cladding modes of the Ex-TFG, enabling stronger absorption in TE mode and more wavelength shift in TM mode. Furthermore, the surrounding refractive index can modulate the complex optical constant of graphene and then the polarization properties of the hybrid waveguide, such as resonant wavelength and peak intensity. Therefore, the unique polarization tuning property induced by the integration of graphene layer with Ex-TFG may endow potential applications in all-in fiber modulator, fiber laser and biochemical sensor. (C) 2016 Optical Society of America

OCIS codes: (060.2340) Fiber optics components; (060.3735) Fiber Bragg gratings; (130.5440) Polarization-selective devices.

http://dx.doi.org/10.1364/OL.99.099999

Optical polarization modulation is one of most crucial operations in a variety of photonics and optoelectronics applications [1], such as optical communication, environmental monitoring, biochemical sensor, etc.. In recent years, graphene as a perfect two-dimensional (2D) material has attracted a great deal of attentions due to its remarkable intrinsic electronic transport properties [2], and has been widely used for numerous photonics and optoelectronic devices [1, 3-5]. Furthermore, relying on its high-carrier mobility, zero-bandgap and complex refractive index characteristics, graphene exhibits broadband and tunable absorptions with wavelength-independent, strong polarization-dependent effects and other optical properties [3, 4], and has been revealed in sandwiched dielectric structure [6], integrated silicon wire waveguide [7] and pump-probe experiments [8, 9]. However, most applications in planar structure/waveguide-coupled graphene optoelectronic devices require the enhanced light-matter interaction such as absorption, and optical fiber with evanescent field leaking out is a promising alternative approach, which provides sufficient interaction length, compared with the extremely limited length (only thickness of graphene) for normal incident light in conventional free-space optical system [10]. Already, the guiding, coupling and polarizing of light in microfibers, side-polished (D-shape) fibers and etched fiber grating structures, have been manipulated to achieve the all-optical modulators [11], switches [12], polarizers [4], sensors [13], etc.

In this letter, we report an integration of few-layer graphene onto an excessively tilted fiber grating (Ex-TFG), which promises unique polarization tuning characteristics. With distinctive core-tocladding mode coupling merits, the Ex-TFG not only provides an accessible evanescent field to enhance light-graphene interaction, but also has two sets of polarization-dependent cladding modes. More interestingly, the strong and broadband light-matter interaction in Ex-TFG-to-graphene waveguide can be remarkably modulated by the surrounding refractive index (SRI). Thus, with exploitation of the polarization sensitive leaky cladding modes enabled by the graphene, the polarization evolution of Ex-TFG demonstrates quite different features in peak intensity change and resonant wavelength shift. Therefore, compared to microfiber and side-polished fiber, the proposed graphene-integrated Ex-TFG with more special polarization dependence and robust structure is a promising alternative and expected to apply in the polarization modulator, fiber laser, and bio-chemical sensor applications.

When the tilt angle of tilted fiber grating is larger than a certain value $\left(\sim 65^{\circ}\right)$, the forward-propagating core mode will be coupled into forward-propagating cladding modes according to phasematch condition [14]. Due to the largely tilted fringes $\left(>80^{\circ}\right)$ induced birefringence in the fiber core, the Ex-TFG shows 
noticeable multi-notch dual-peak feature, i.e. the resonant peaks of orthogonal TE and TM cladding modes. The resonant wavelength $\lambda_{i, \text { clad }}^{m}$ of the $m$ th cladding mode for the Ex-TFG, is determined by the phase-match condition and can be expressed as follows

$$
\lambda_{i, \text { clad }}^{m}=\left[n_{\text {eff,core }}(T, \varepsilon)-n_{i, \text { eff,clad }}^{m}\left(n_{\text {SRI }}, T, \varepsilon\right)\right] \Lambda_{\mathrm{g}}(T, \varepsilon) / \cos \theta \text {. (1) }
$$

Where, $i$ represents TE or TM, $n_{\text {eff,core }}$ and $n_{i, \text { eff,clad }}^{m}$ are the effective indices of the core mode and the $m$ th cladding mode, respectively, $\Lambda_{\mathrm{g}}$ is the nominal grating period, $\theta$ is the tilt angle, and $T, \varepsilon, n_{\mathrm{SRI}}$ are the temperature, strain, and refractive index of surrounding medium, respectively. The resonant wavelengths of TE and TM cladding modes with the same order could have the separation depending on the difference of their effective indices. From Eq. 1, apart from the temperature and strain, we see the environmental condition can affect the effective indices of the cladding modes, and thus the resonant wavelengths.

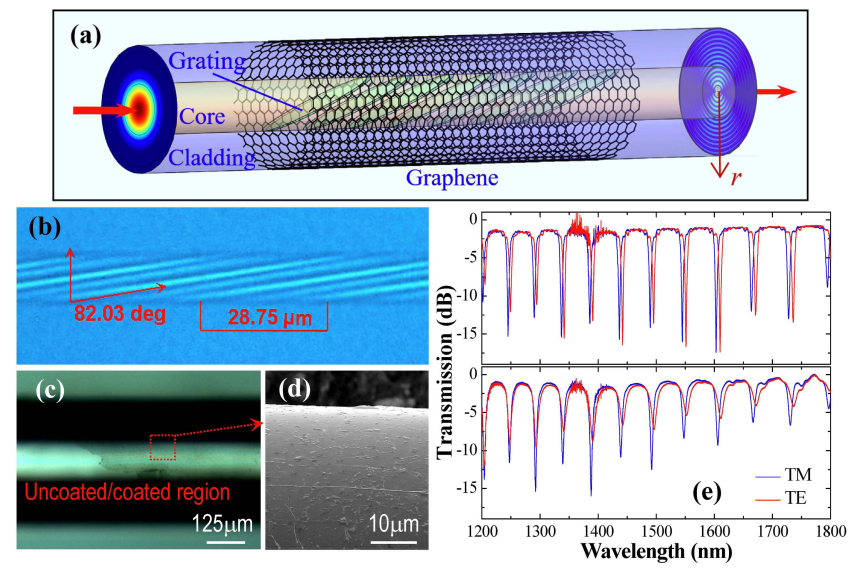

Fig. 1. (a) Schematic model of Ex-TFG-to-graphene hybrid waveguide, optical microscopic images of (b) tilted fringes of Ex-TFG and (c) graphene-coated Ex-TFG, (d) SEM image of the Ex-TFG surface, and (f) transmission spectra of the Ex-TFG at two orthogonal polarizations without (upper) and with (lower) graphene layer.

When the few-layer graphene with complex refractive index is integrated onto the Ex-TFG surface to form an Ex-TFG-to-graphene hybrid waveguide, as schematically shown in Fig. 1(a) , the lightmatter interaction in two polarizations will induce different coupling behavior, exhibiting the variation of spectral intensity and resonant wavelength attributed to differential effect of graphene on the two polarized modes $[4,6,7]$. The employed Ex-TFGs were UV inscribed in hydrogenated SMF-28 fiber by using the scanning phase-mask technique and a frequency doubled continuous wave (CW) argon-ion laser. The excessively tilted structure was realized by rotating the phase mask in the fabrication system [14]. Figure 1(b) shows a microscopic image of the tilted fringes at $\sim 82^{\circ}$ in the fiber core by a high-resolution microscope (Carl Zeiss Axiotron 2). The graphene film used was grown on copper foils using chemical vapor deposition and had a five-layer thickness on each side of the foil to provide stronger interaction with the evanescent field than the monolayer graphene $[12,15]$. The number was distinguished by a transmission electron microscope and Raman spectroscopy [16]. Copper foil was then chemically removed, leaving double fivelayer and $\sim 12 \mathrm{~mm}$ long graphene film afloated on top of water and then transferred around the optical fiber [17]. Figures 1(c) and 1(d) display optical microscopic and scanning electron microscopy (SEM) images of the graphene-coated Ex-TFG surface, showing a clear edge of the uniform graphene layer around the fiber. The polarization dependence of Ex-TFG was tested by using an experimental system, including super-broadband light source, infiber linear polarizer, polarization controller, and optical spectrum analyzer (OSA). Launched from the light source, the light is polarized and adjusted by the polarizer and the corresponding controller. During the adjustment of the polarization, we recorded the transmission spectra of Ex-TFG at two orthogonal polarizations before and after graphene integration, as depicted in Fig. 1(e). We can observe a set of comblike resonances with two polarizations, and the resonant intensity and polarization dependence are wavelength/mode-order dependent.
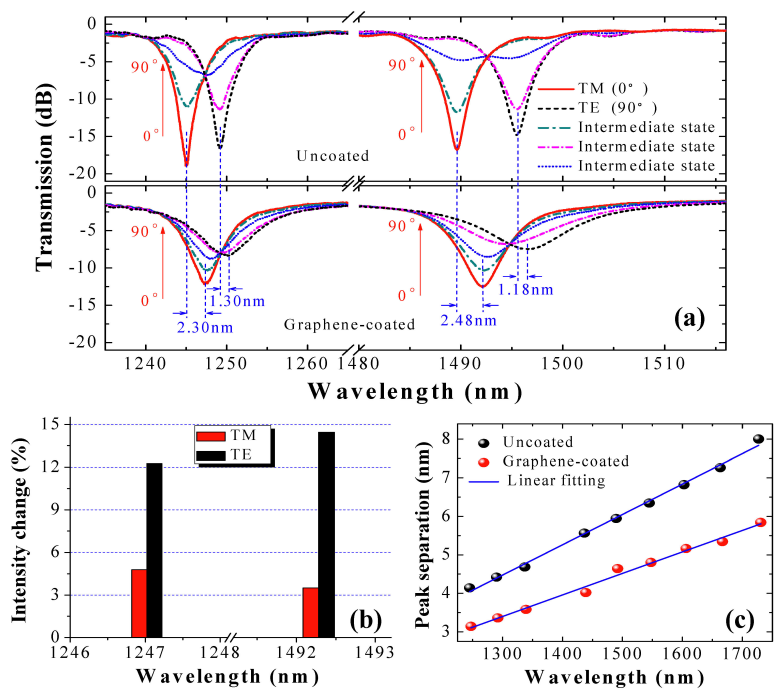

Fig. 2. (a) Transmission spectra of Ex-TFG obtained by adjusting the azimuth angle of polarization controller before and after graphene coating, (b) variations of the coupling intensities of the resonant peaks at shorter and longer wavelengths, and (c) dual-peak separation between two orthogonal polarizations at different resonant peaks.

In the detailed examination, we selected two cladding modes, one at shorter wavelength $(\sim 1250 \mathrm{~nm})$ and another at longer wavelength $(\sim 1500 \mathrm{~nm})$, to investigate the effect of graphene layer on broadband polarization dependence. For the two cladding mode resonances of the uncoated Ex-TFG, measured in two zoomed ranges, as depicted at the top of Fig. 2(a), we can clearly see the TM peaks gradually disappears, some intermediate states appears, and finally the TE ones grow to a strong peak of $\sim 17-\mathrm{dB}$ attenuation when the polarization of the launched light changing from $0^{\circ}$ to $90^{\circ}$. After the graphene integration, the leaky modes radiated from the cladding are key signatures of strong enhancement of evanescent field by graphene in terms of coupling and absorption. The significant spectral attenuation of two polarization peaks resulted from the interaction of the evanescent field and graphene can be clearly observed from the lower part of Fig. 2 (a), indicating effective coupling between the Ex-TFG and graphene. By examining the spectral evolution under different polarizations, it was found that the TE light from the cladding modes of all orders is more coupled into graphene layer, which attests to polarization-sensitive absorption of graphene for all leaky modes $[3,4]$. It is apparent that the change of coupling 
intensity of TE polarization is always larger than that of TM for all cladding modes [18], as shown in Fig. 2(b). However, the variations of spectral properties are mode-order independent, i.e. both TM and TE peaks show similar manner at different wavelengths. Also, after integrating with graphene, the resonant wavelengths of TM modes showed more pronounced red-shift (2.30/2.48nm at shorter/longer wavelengths, respectively) than that of TE modes $(1.30 / 1.18 \mathrm{~nm}$ at shorter/longer wavelengths, respectively), which demonstrates larger change to effective index of TM modes than that of TE modes. For the uncoated Ex-TFG, the polarization effect induced spectral separations between the dual peaks at shorter and longer wavelength are $4.14 \mathrm{~nm}$ and $5.94 \mathrm{~nm}$, respectively, while, after graphene coating, the separations reduced to $3.14 \mathrm{~nm}$ and $4.64 \mathrm{~nm}$, due to the different effects of graphene on effective indices of two polarization modes. As shown in Fig. 2(c), with the resonant wavelength increase, the separation linearly increases, indicating wavelength dependent feature, which can be explained by the increasing effective index difference between the TM and TE modes [18].
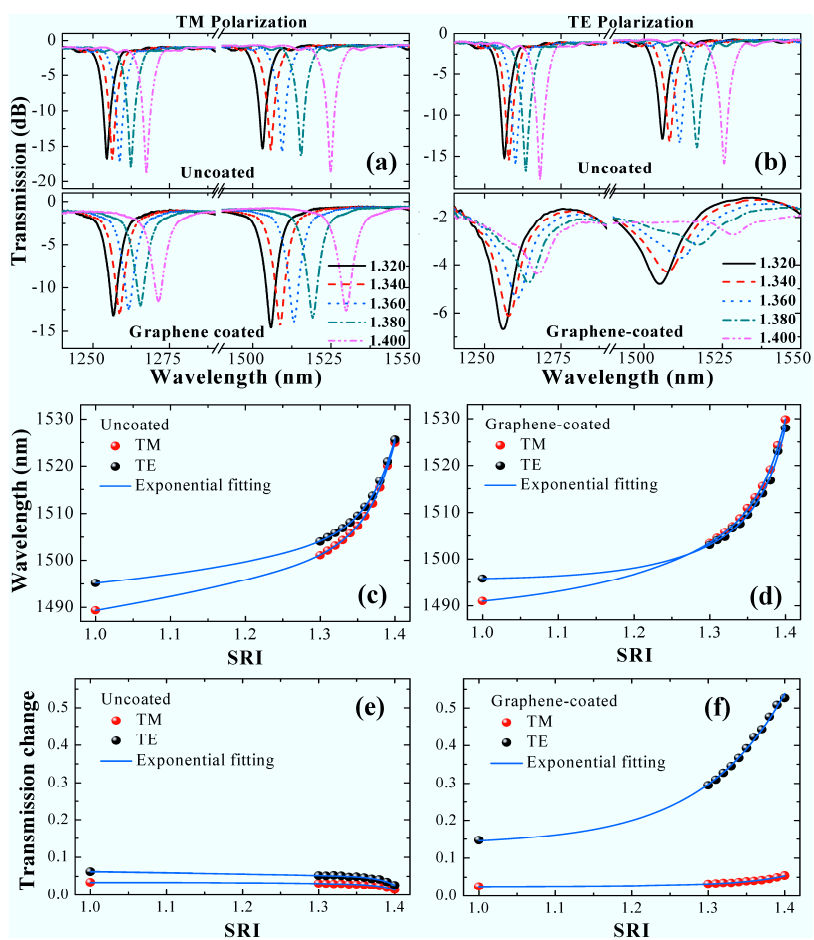

Fig. 3. Tuning characteristics of polarization of the Ex-TFG without and with graphene coating in SRI solutions: (a) and (b) spectral evolutions, (c) and (d) wavelength shifts, and (e) and (f) transmission change of the TM and TE peaks at longer wavelength.

The tuning characteristics of polarization of the Ex-TFG cladding modes with SRI variation were experimentally examined by using a series of refractive index liquids (from Cargille Labs) ranging from 1.300 to 1.400 with interval of 0.01 . With the graphene sandwiched between index gel and fiber cladding, the modal field distribution in these three layers is different for TM and TE modes, providing their different propagation behaviors. Meanwhile, different intensity components are also dependent on the change of SRI, since it can modulate the effective index of cladding mode. The experiment results are shown in Fig. 3 for two polarization components before and after graphene coating. For the uncoated Ex-TFG, the spectral variation including the peak wavelength and intensity shows similar manner for TM and TE peaks, and the SRIinduced coupling coefficient variation results in small increase of the peak intensity, as shown in Figs. 3(a) and 3(b) top plots, and Fig. 3(e). However, for the graphene-coated Ex-TFG, their spectra show completely different evolutions, especially in the coupling intensity due to the polarization-sensitive absorption caused by relatively larger imaginary part in TE mode, as shown in Figs. 3(a) and 3(b) bottom plots. Although the peaks at shorter and longer wavelengths present a similar wavelength shift trend with the change of the SRI, the peak at longer wavelength is more sensitive to SRI than that at the shorter wavelength. For clarity, we only plotted the peak shift at longer wavelength, as shown in Figs. 3(c) and 3(d). From the peak wavelength shifts of TM and TE modes, we can obtain that the SRI sensitivity of the former is slightly higher than that of the latter. From Fig. 3(c), we can see for the uncoated Ex-TFG, the separation between the dual-peak reduces with increasing of SRI, finally merging to form one. However, after wrapping graphene around Ex-TFG, with increasing of SRI, shifting of peaks for TM modes is faster than that of TE mode, making them surpassing TE mode at certain SRI. In addition, quantitatively, due to the presence of graphene layer, the SRI response coefficients of $\mathrm{TM}$ and TE resonant wavelengths increase by $10.6 \%(9.2 \%)$ and $7.9 \%(5.7 \%)$ at shorter (longer) wavelengths, respectively.

Compared with the uncoated sample, the spectral evolution of graphene-coated Ex-TFG clearly shows a gradual "disappearance" and "spreading" for the TE modes in the SRI range from 1.000 1.400. In Figs. 3(e) and 3(f), we exploited the transmission change to present the absorption amount of light by graphene. It can be clearly seen that the graphene layer has more absorption on TE modes, making it too lossy to build strong resonance along the grating. As a consequence, the coupling intensity change of TE mode indicates $>13$ times larger than that of TM mode.

In addition, it is worth to mention that the spectra of both uncoated and graphene-coated Ex-TFGs can be reverted back to their original position in air after each SRI examination, and show the same spectral attenuation and wavelength shift for the same solution. Thus both uncoated and graphene-coated Ex-TFGs exhibit excellent repeatability and stability.

In order to obtain further insight, we theoretically analyzed and numerically solved the complex vector modes in such a waveguide structure shown in Fig. 1(a) to explore the polarization coupling properties of cladding mode by using improved full-vector finitedifference method [19]. In the simulation model, a 4nm thick graphene layer was placed around the standard single-mode fiber (SMF) Ex-TFG, and the graphene's refractive index was calculated according to the intraband and interband conductivities $\sigma(\omega)=\sigma_{\text {intra }}(\omega)+\sigma_{\text {inter }}(\omega)$ described in Refs. $[4,12,20]$. Figure 4(a) shows radial distributions of TM and TE modal fields of the highorder (28th-order as an example) cladding mode at wavelength of $1.5 \mu \mathrm{m}$ in Ex-TFG structure. From the insets of Fig. 4(a), in the presence of graphene layer, we can clearly see that the modal intensity of TE mode decreases more than that of TM mode in the fiber core region. This means that the graphene reduces the coupling between the core and cladding modes, especially in TE polarization, leading to a decrease of resonant peak. The intensity difference $I_{\mathrm{g}}-I_{\mathrm{b}}$ of modal fields between graphene-coated and uncoated (bare) Ex-TFG are depicted in Fig. 4(b), more clearly, 
manifesting a larger intensity modification for the TE mode than TM mode, which is consistent with experimental results shown in Figs. 2(a) and 2(b).
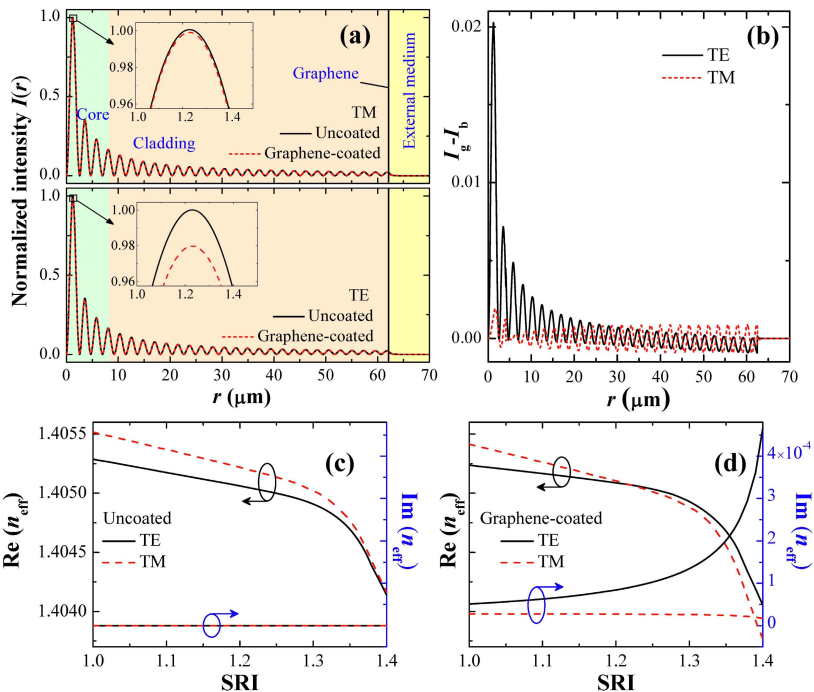

Fig. 4. (a) Radial distributions (with the zoomed distribution in fiber core) of simulated TM (upper) and TE (lower) cladding modes at the wavelength of $1.5 \mu \mathrm{m}$, (b) intensity differences of modal fields between graphene-coated and uncoated Ex-TFG for TE and TM modes, variations of real and imaginary parts of effective index of the cladding mode of (c) uncoated and (d) graphene-coated Ex-TFG with the SRI.

Figures 4(c) and 4(d) depict the changes of the real part $\operatorname{Re}\left(n_{\text {eff }}\right)$ (induces the resonant wavelength change) and imaginary part $\operatorname{Im}\left(n_{\text {eff }}\right)$ (induces the absorption change) of the effective index of cladding mode with the increase of SRI. For the uncoated Ex-TFG, $\operatorname{Re}\left(n_{\text {eff }}\right)$ of TM mode is invariably higher than that of TE mode, and they will tend to be the same when the SRI approaching 1.4. As a result, with the increasing SRI, the resonant wavelength of TM mode will exhibit a larger red-shift than that of TE one according to Eq. 1. However, for graphene-coated Ex-TFG, SRI-induced effective index $\operatorname{Re}\left(n_{\text {eff }}\right)$ of TM mode falls faster than that of TE mode, leading to a cross point at SRI of $\sim 1.22$ in $\operatorname{Re}\left(n_{\text {eff }}\right)$ vs. SRI curves. After the cross point, the positions of resonant wavelengths of TE and TM modes are reversed. Therefore, from Eq. 1 and simulation results, the decreasing $\operatorname{Re}\left(n_{\text {eff }}\right)$ with the SRI can explain the red-shift of the resonant wavelength from the experimental observations in Figs. 3(c) and 3(d), and the experimental measurements follow the same trends as simulation results. And also, the total change amout of $\operatorname{Re}\left(n_{\text {eff }}\right)$ of cladding mode of graphene-coated Ex-TFG is larger than that of uncoated one in the SRI range of 1.000 1.400, which can explain the phenomenon that the SRI responses of resonant wavelengths have a certain enhancement after graphene coating. In the meantime, from Fig. 4(d), compared with the uncoated sample, $\operatorname{Im}\left(n_{\text {eff }}\right)$ of TM mode of graphene-coated Ex-TFG has a nonzero value and relatively small magnitude than that of the TE one, representing more absorption of graphene layer on TE mode, and further causing more transmission change of TE peak, as also displayed in Fig. 3(f). From our simulation results, we can therefore reveal that the resonant wavelength and intensity of two polarizations will have different evolution behaviors, as a result of the direct consequence of the different variations in real and imaginary parts of complex refractive index of graphene.
In conclusion, we have studied the interaction of electromagnetic field with Ex-TFG structures and revealed the unique polarization tuning properties in an Ex-TFG-to-graphene hybrid waveguide. The polarization-dependent transmission of broad spectral range in the hybrid waveguide has been demonstrated. Numerical calculations and experimental results reveal the polarization tunable effect originates from polarizationsensitive coupling between the graphene and the Ex-TFG cladding mode. The polarization-dependent resonant wavelength shift and intensity attenuation are environment-sensitive, as a result of the modulation of complex optical constant of graphene by SRI. The broadband polarizing effect of the proposed graphene-integrated Ex-TFG provides multi-wavelength light modulation, with added advantages of more robust structure and easier fabrication than microfiber and side-polished fiber structure. In addition, the polarizing effect can be further modulated by adjusting the tilted angle, grating period, and core/cladding diameter of Ex-TFG to satisfy actual wavelength range and extinction ratio requirements.

Funding. Marie Skłodowska-Curie Individual Fellowships in the European Union's Horizon 2020 Research and Innovation Programme (660648); National Natural Science Foundation of China (NSFC) (61505165); Natural Science Basic Research Plan in Shaanxi Province of China (2016JQ6032).

\section{References}

1. Z. Sun, A. Martinez, and F. Wang, Nat. Photon. 10, 227 (2016).

2. F. Bonaccorso, Z. Sun, T. Hasan, and A. C. Ferrari, Nat. Photonics 4,611 (2010).

3. F. Xing, Z.-B. Liu, Z.-C. Deng, X.-T. Kong, X.-Q. Yan, X.-D. Chen, Q. Ye, C.-P. Zhang, Y.-S. Chen, and J.-G. Tian, Scientific Reports 2, 908 (2012).

4. Q. Bao, H. Zhang, B. Wang, Z. Ni, C. H. Y. X. Lim, Y. Wang, D. Y. Tang, and K. P. Loh, Nat. Photon. 5, 411 (2011).

5. X. Gan, R.-J. Shiue, Y. Gao, I. Meric, T. F. Heinz, K. Shepard, J. Hone, S. Assefa, and D. Englund, Nat. Photon. 7, 883 (2013).

6. Q. Ye, J. Wang, Z. Liu, Z.-C. Deng, X.-T. Kong, F. Xing, X.-D. Chen, W.-Y. Zhou, C.-P. Zhang, and J.-G. Tian, Appl. Phys. Lett. 102, 021912 (2013).

7. K. Rai, T. Shinichi, T. Tai, W. Kaori, S. Satoru, H. Hiroki, N. Hirochika, and Y. Koji, Japanese Journal of Applied Physics 52, 060203 (2013).

8. M. Mittendorff, T. Winzer, E. Malic, A. Knorr, C. Berger, W. A. de Heer, H. Schneider, M. Helm, and S. Winnerl, Nano Lett. 14, 1504 (2014).

9. J. Yao, X. Zhao, X.-Q. Yan, C. Gao, X.-D. Chen, W. Xin, Y. Chen, Z.-B. Liu, and J.-G. Tian, Optical Materials Express 5, 1550 (2015).

10. J.-L. Kou, J.-H. Chen, Y. Chen, F. Xu, and Y.-Q. Lu, Optica 1, 307 (2014).

11. W. Li, B. Chen, C. Meng, W. Fang, Y. Xiao, X. Li, Z. Hu, Y. Xu, L. Tong, H. Wang, W. Liu, J. Bao, and Y. R. Shen, Nano Lett. 14, 955 (2014).

12. X. Gan, C. Zhao, Y. Wang, D. Mao, L. Fang, L. Han, and J. Zhao, Optica 2, 468 (2015).

13. Y. Wu, B. Yao, A. Zhang, Y. Rao, Z. Wang, Y. Cheng, Y. Gong, W. Zhang, Y. Chen, and K. S. Chiang, Opt. Lett. 39, 1235 (2014).

14. K. Zhou, L. Zhang, X. Chen, and I. Bennion, J. Lightwave Technol. 24, 5087 (2006).

15. R. R. Nair, P. Blake, A. N. Grigorenko, K. S. Novoselov, T. J. Booth, T. Stauber, N. M. R. Peres, and A. K. Geim, Science 320, 1308-1308 (2008).

16. M. Qi, Y. Zhou, F. Hu, X. Xu, W. Li, A. Li, J. Bai, and Z. Ren, J. Phys. Chem. C 118, 15054 (2014).

17. B. Jiang, X. Lu, X. Gan, M. Qi, Y. Wang, L. Han, D. Mao, W. Zhang, Z. Ren, and J. Zhao, Opt. Lett. 40, 3994 (2015).

18. Z. Yan, H. Wang, C. Wang, Z. Sun, G. Yin, K. Zhou, Y. Wang, W. Zhao, and L. Zhang, Opt. Express 24, 12107 (2016).

19. G. Yin, S. Lou, Q. Li, and H. Zou, Opt. Laser Technol. 48, 60 (2013).

20. S. A. Mikhailov and K. Ziegler, Physical Review Letters 99, 016803 (2007). 